\title{
Anticholinergic Drugs in Geriatric Psychopharmacology
}

\author{
Jorge López-Álvarez ${ }^{1,2}$, Julia Sevilla-Llewellyn-Jones ${ }^{3,4}$ and Luis Agüera-Ortiz ${ }^{1,2,5,6 *}$ \\ ${ }^{1}$ Servicio de Psiquiatría, Hospital 12 de Octubre (imas12), Madrid, Spain, ${ }^{2}$ Instituto de Investigación, Hospital 12 de Octubre \\ (imas12), Madrid, Spain, ${ }^{3}$ Instituto de Psiquiatría y Salud Mental, Hospital Clínico San Carlos, Madrid, Spain, ${ }^{4}$ Instituto \\ de Investigación (IdISSC), Hospital Clínico San Carlos, Madrid, Spain, ${ }^{5}$ Centro de Investigación Biomédica en Red de Salud \\ Mental, Ministry of Science and Innovation, Madrid, Spain, ${ }^{6}$ Departamento de Medicina Legal, Psiquiatría y Patología, \\ Facultad de Medicina, Universidad Complutense de Madrid, Madrid, Spain
}

\section{OPEN ACCESS}

Edited by: Angel L. Montejo, University of Salamanca, Spain

Reviewed by:

Byron Creese,

University of Exeter, United Kingdom Bashkim Kadriu,

National Institute of Mental Health (NIMH), United States

*Correspondence:

Luis Agüera-Ortiz

laguera@med.ucm.es

Specialty section:

This article was submitted to Neuropharmacology,

a section of the journal

Frontiers in Neuroscience

Received: 07 August 2019 Accepted: 22 November 2019 Published: 06 December 2019

Citation:

López-Álvarez J,

Sevilla-Llewellyn-Jones $J$ and Agüera-Ortiz L (2019) Anticholinergic Drugs in Geriatric

Psychopharmacology.

Front. Neurosci. 13:1309. doi: 10.3389/fnins.2019.01309
Drugs with anticholinergic action are widely prescribed in the elderly population due to their potential clinical benefits. However, these benefits are limited by adverse effects which may be serious in particular circumstances. This review presents different aspects of the use of anticholinergics in old age with a focus in psychogeriatric patients. We critically review published data on benefits and disadvantages of anticholinergics, which are often controversial. Prevalence, pathophysiology and measurement methods of the anticholinergic action of drugs are discussed. We also present the most important drawbacks resulting from its use, including effects on cognition in healthy and cognitively impaired people, in aged schizophrenia patients, emergence of delirium and psychiatric symptoms, influence in functionality, hospitalization, institutionalization and mortality, and the potential benefits and limitations of their discontinuation. Finally, we suggest practical recommendations for the safe use of anticholinergics in clinical conditions affecting elderly patients, such as dementia, schizophrenia and acute hallucinatory episodes, depression, anxiety, Parkinson's disease, cardiovascular conditions and urinary incontinence.

Keywords: anticholinergic drugs, elderly, potentially inappropriate prescriptions, adverse effects, cognition, dementia

\section{INTRODUCTION}

Anticholinergic drugs bind to the muscarinic receptors and block acetylcholine neurotransmission, which is involved in many major body functions including central nervous system (CNS) functions such as attention, learning and memory mechanisms and peripheral nervous system (PNS) actions which are related to basal functioning of the organism such as urination, intestinal transit or heart rhythm regulation. Drugs with anticholinergic action may bind exclusively to muscarinic receptors or may also bind to other receptors with agonist or antagonist actions, and therefore, have different therapeutic targets and denominations (e.g., tricyclic antidepressants).

Since cholinergic transmission is involved in many physiological functions, anticholinergic drugs can have adverse effects and affect the central and peripheral nervous systems. On the one hand, adverse effects on the central nervous system (CNS) include dysfunction in different cognitive domains, cognitive impairment, an acceleration of neurodegenerative processes, the appearance of psychotic or confusional symptoms and functionality disturbances. On the other hand, adverse effects on the PNS include dry mouth, urinary retention, constipation and paralytic ileus, increased heart rate and blurred vision, among others. 


\section{ANTICHOLINERGIC DRUGS}

Anticholinergic drugs are used for multiple medical conditions, such as urinary dysfunction, peptic ulcer disease, irritable bowel syndrome or Parkinson's disease. Moreover, anticholinergics are frequently used as anesthetic agents, and for neurologic and psychiatric conditions (Potamianos and Kellett, 1982). While some anticholinergic drugs are well known and their anticholinergic effects are fully recognized, other drugs have less known anticholinergic activity (AA). Table 1 shows the most frequently used neuropsychiatric drugs with anticholinergic effects and Table 2 some commonly prescribed non-psychoactive drugs with anticholinergic effects.

The main properties of anticholinergic drugs are related to their action on central and/or peripheral cholinergic receptors (Lechevallier-Michel et al., 2005). These actions can cause symptoms such as cognitive disorders, which can be mistakenly assumed as part of normal manifestations of aging (Kersten et al., 2013).

It is also important to note that some drugs, such as amoxicillin, diazepam, digoxin, duloxetine, fentanyl, furosemide, lansoprazole, metformin, phenytoin or topiramate only have AA at high doses (Chew et al., 2008). Furthermore, the combination of different drugs (or even nutritional supplements such as chondroitin or some vitamins) can cause or exacerbate the adverse events of an already prescribed anticholinergic drug.

Anticholinergics have many side effects and depend on the anticholinergic drug load and individual vulnerability. Table 3 summarizes the side effects of anticholinergic agents attending to the anticholinergic load.

Due to their side effects, the prescription of anticholinergic drugs may be considered inappropriate in certain circumstances. Potentially inadequate anticholinergic drug use in older adults is included in the Beers (American Geriatrics Society 2012 Beers Criteria Update Expert Panel, 2012) and the STOPP-START criteria (O’Mahony et al., 2015). However, for certain clinical syndromes the benefits of anticholinergics are greater than their risks, and their prescription can be considered adequate in the elderly in some cases (Table 4).

\section{PATHOPHYSIOLOGY OF ANTICHOLINERGIC DRUGS IN THE BRAIN}

There are five subtypes of muscarinic receptors, M1 to M5. M1, M2 and M4 are, to date, muscarinic receptors that appear to be more related to the possible anticholinergic deleterious effects. M1 receptors are the most common in the CNS and have a crucial role related to executive functions and episodic memory in the prefrontal cortex and hippocampus, respectively (Sathienluckana et al., 2018). M2 receptors participate in memory processing and M4 receptors regulate acetylcholine levels. The antagonism of these receptors can lead to cognitive disturbance and cell death (Bishara et al., 2017). In fact, alteration of cholinergic function affects gating of sensory input and information processing,
TABLE 1 | Neuropsychiatric drugs with anticholinergic side effects.

High anticholinergic load

Tryciclicantidepressants

Amitryptiline

Clomipramine

Desipramine

Imipramine

Antipsychotics

Chlorpromazine

Clozapine

Fluphenazine

Loxapine

Olanzapine

Perphenazine

Antiepileptics

Carbamazepine

Oxcarbazepine

Anticholinergics as such

Benztropine

Biperiden

Trihexyphenidyl

Low-moderate anticholinergic load

Antidepressants

Fluoxetine

Fluvoxamine

Mirtazapine

Nortriptiline

Paroxetine

Sertraline

Trazodone

Antipsychotics

Haloperidol

Quetiapine

Ziprasidone

Benzodiazepines

Alprazolam

Chlordiazepoxide

Clonazepam Clorazepate

Diazepam

Flurazepam

Lorazepam

Midazolam

Antiepileptics

Valproate

Antiparkinsonians

Amantadine

Bromocriptine

Carbidopa- Levodopa

Pramipexol

Selegiline

Opioids

Codeine

Fentanyl

Morphine

Tramadol 
TABLE 2 | Common non-psychoactive drugs with anticholinergic effects.

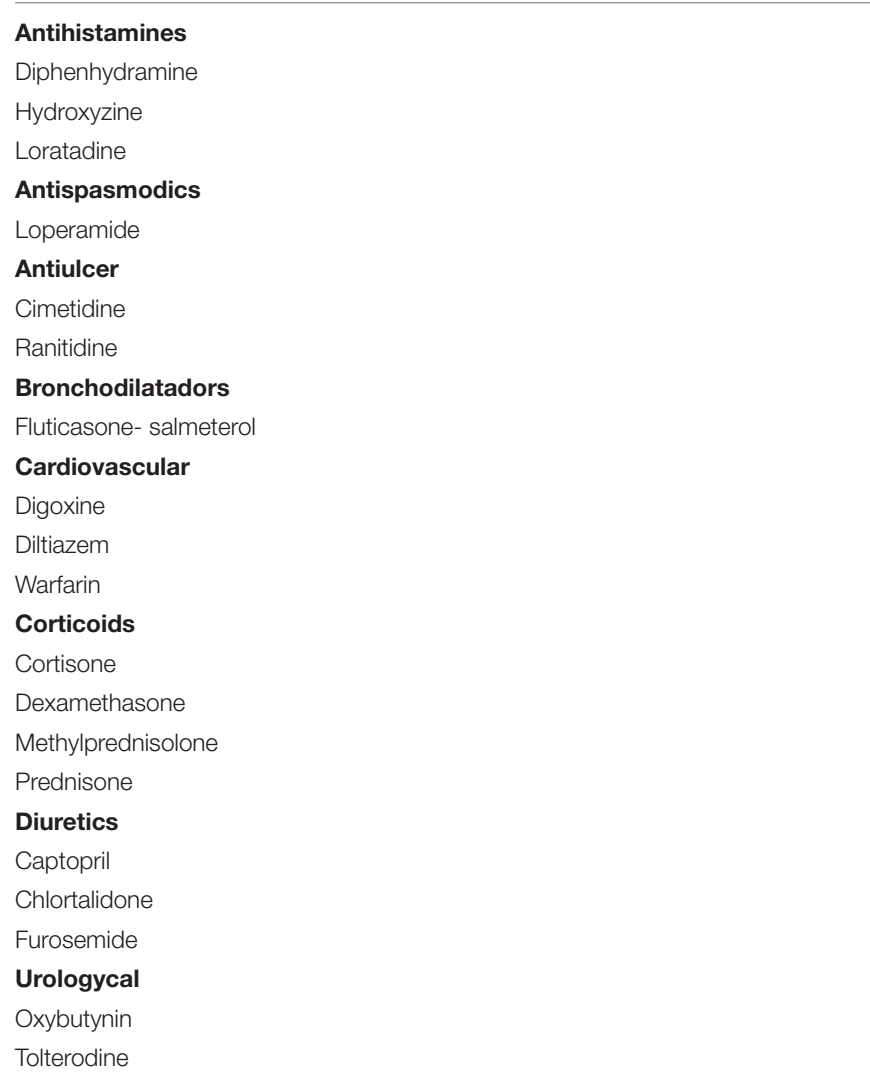

TABLE 3 | Anticholinergic side effects of drugs.

\begin{tabular}{ll}
\hline $\begin{array}{l}\text { Mild or moderate anticholinergic } \\
\text { effects }\end{array}$ & Severe anticholinergic effects \\
\hline Somatic symptoms & Somatic symptoms \\
Anhidrosis & Congestive heart failure \\
Blurry vision & Fecal impaction/paralytic ileus \\
Constipation & Malnutrition \\
Dry mouth & Respiratory infections \\
Fatigue & Tachyarrhythmia \\
Mydriasis & Urinary retention/urinary tract infection \\
Tachycardia/palpitations & Cardiac attack \\
Urinary hesitancy & \\
Neuropsychiatric symptoms & Neuropsychiatric symptoms \\
Drowsiness & Agitation \\
Nervousness, excitement & Ataxia \\
Mild amnesia and cognitive dysfunction & Complex visual hallucinations \\
Poor attention & Delirium \\
Restlessness & Epileptic seizures \\
& Hallucinations \\
& Hyperreflexia \\
& Nocturnal rhythm disturbance \\
\hline
\end{tabular}

prerequisites for learning and memory. Cognitive effects depend on anticholinergic load, baseline cognitive function and individual pharmacokinetics and pharmacodynamics
TABLE 4 | Clinical problems in the elderly population where the prescription of anticholinergics may be appropriate or inevitable in certain circumstances.

\begin{tabular}{ll}
\hline Psychiatric conditions & Non-psychiatric conditions \\
\hline $\begin{array}{ll}\text { Primary psychotic disorders (schizophrenia, } \\
\text { schizoaffective disorder, delusional disorder) }\end{array}$ & Gastroesophageal reflux disease \\
Bipolar disorder & Cardiovascular diseases \\
Endogenous depression & Irritable bowel syndrome \\
Psychotic depression & Muscle spasms/low back pain \\
Obsessive-Compulsive disorder & Neuropathic pain \\
Severe anxiety disorders & Urinary incontinence (after \\
& non-pharmacological approach) \\
Severe insomnia & \\
Drug-induced acute dystonia & \\
Secondary parkinsonism &
\end{tabular}

variability, and are also influenced by metabolism alterations (Roe et al., 2002).

Anticholinergic drugs only produce central effects if crossing the blood-brain barrier. This is less likely if the drug is a substrate of the $P$-glycoprotein multi-drug efflux pump (Bishara et al., 2017) and more likely if the activity of that transporter is reduced. In this line, it is important to note that bloodbrain barrier permeability is increased in people with diabetes or multiple sclerosis, and by drugs such as lansoprazole, omeprazole, loperamide, simvastatin, clonidine, or methyldopa.

Long-term continuing therapy with anticholinergic drugs could induce brain changes partially comparable to those present in Alzheimer's disease. In fact, cognitive dysfunction in $\mathrm{AD}$ is related with different aspects, such as decrease in the number of cholinergic neurons, acetylcholine receptor dysfunctions, and signaling dysregulation. Decline severity is proportional to the alteration of the cholinergic system (Swami et al., 2016). In this line, an anatomopathological study found that amyloid plaques were 2.5 times more frequent and neurofibrillary tangles were increased in patients with Parkinson's disease treated with anticholinergic drugs in the long term compared to short-term treated or non-treated patients (Perry et al., 2003). However, these findings were not confirmed in a similar study (Gray et al., 2018). Interestingly, Caccamo et al. (2006) found that long-term cholinergic receptor blockade increased the beta-amyloid peptide in the cortex, hippocampus and amygdala.

Using neuroimaging techniques, a cross-sectional study in older adults found an association between moderate or high anticholinergic load and reduced cerebral metabolism, decreased cortical volume and thickness of the temporal cortex, increased ventricular volume, temporal lobe atrophy and global atrophy (Risacher et al., 2016). Accordingly, a longitudinal study detected higher rates of atrophy in the total cerebral volume and in the gray matter associated to anticholinergic drug consumption, finding a dose-dependent relationship between anticholinergic load and cortical thinning (Chuang et al., 2017). A different study linked the integrity of the cholinergic system with the correct functioning of episodic memory (Richter et al., 2014). Additionally, Sperling et al. (2002) found that the administration of 
scopolamine, a potent anticholinergic drug, decreased brain activation in the hippocampus, fusiform gyrus and lower frontal regions, and was associated with lower performance in neuropsychological tests.

The concept of "anticholinergic spectrum disorders" has been proposed by Hori et al. (2016), which would include Alzheimer's disease, Lewy body dementia and delirium. They consider that acetylcholine downregulation causes an endogenous AA cascade that can exacerbate neurodegeneration (Kitajima et al., 2015). This symptomatology can be precipitated by a moderate and asymptomatic cholinergic deficit in the presence of "AA inserts" such as polypharmacy, physical illnesses or stress, and is reversible when the action of these "AA inserts" disappears. However, when endogenous AA appears, irreversible progression to dementia occurs (Konishi et al., 2015). In relation to this, mice experiments showed that a greater anticholinergic action increased tau pathology and its insolubility, which could be reversed with the use of procholinergic drugs (Yoshiyama et al., 2015). In fact, acetylcholinesterase inhibitors (AchI) increase cholinergic activity, and although they do not halt disease progression, they are currently considered the treatment of choice for dementias presenting with cholinergic deficit, such as Alzheimer's disease, Lewy body dementia or Parkinson's dementia.

Additionally, psychotic symptoms in Alzheimer's disease have been explained by a central cholinergic deficiency syndrome that would cause dysfunction in detecting, selecting, discriminating and processing sensory inputs (Lemstra et al., 2003). Thus, psychosis is associated with metabolic and perfusion alterations in the temporal and frontal cortices, where the cholinergic deficit is greater (Cummings and Back, 1998). Psychotic symptoms in Alzheimer's disease are also associated with elevated binding to M2 receptors at frontal and temporal cortical level (Lai et al., 2001).

\section{PREVALENCE}

Despite the deleterious effects associated to the use of anticholinergic drugs in the elderly, these medications are widely prescribed, mainly because they are efficacious and less harmful alternatives are not available. For example, in Primary Care, a prevalence of $12.5 \%$ of prescriptions was detected in this population. Among these, more than half were for a psychotropic drug (Gorup et al., 2018). In fact, the prevalence increases to $25.8 \%$ if the aged person presents with mnesic complaints (Grande et al., 2017).

Anticholinergic drugs consumption has been associated with lower educational level, chronic comorbidities (Grande et al., 2017), hospitalization or specialist visits in the previous year, urinary incontinence, arterial hypertension (Niznik et al., 2017), impaired health status, anxiety, mood disorders (Kachru et al., 2015) and polypharmacy.

While it has been reported that during hospital admission the number of patients who receive anticholinergics and their related anticholinergic load increases, it is not yet clarified whether this is related to the medical circumstances of the hospitalization or the circumstances around the hospitalization itself (Brombo et al., 2018). However, this increase affects $79 \%$ of the hospitalized patients (Pasina et al., 2019). In addition, around the $20 \%$ of the patients in specialized geriatric hospitalization units -a setting where the use of anticholinergics is often well scrutinized -receive high-potency drugs (Pfistermeister et al., 2017), which supports the idea that in a significant number of cases, this use is not easily avoidable.

In nursing homes, the most prevalent anticholinergics were cardiovascular drugs, followed by antipsychotics and antidepressants ( $\mathrm{Wu}$ et al., 2017). These findings are in line with those of a community-dwelling elderly patients study (Green et al., 2016).

In patients with mild cognitive impairment (MCI) or dementia treated in memory clinics, $44.7 \%$ were taking anticholinergic drugs, with $11.7 \%$ receiving a high anticholinergic load (Cross et al., 2016). Up to half of the patients with MCI have a high anticholinergic load due to the additive effect of several drugs (Green et al., 2016). The prevalence of anticholinergic use is increased in the institutionalized elderly, especially if they are diagnosed with dementia, and even in those with a terminal condition (Williams et al., 2018).

In earlier clinical studies the potentially inappropriate prescription of anticholinergics was not usually taken into consideration (Kachru et al., 2015). Nowadays, the Beers (American Geriatrics Society 2012 Beers Criteria Update Expert Panel, 2012) and the STOPP-START criteria (O'Mahony et al., 2015) consider that their prescription is inappropriate in most cases. However, whether or not prescribing anticholinergics is appropriate is something still unclear (López-Álvarez and Agüera Ortiz, 2019). In fact, a study using the Beers criteria found that $39.9 \%$ of elderly people with dementia on an outpatient basis were taking anticholinergic drugs classified as potentially inadequate (Bala et al., 2019).

Despite their antagonistic effects, the combined use of AchI and anticholinergics is common, especially when depression, chronic obstructive pulmonary disease, hypertension, chronic kidney disease and/or cardiac failure are present (Mantri et al., 2019). This combination is frequently used to treat procholinergic side effects, such as nausea, dizziness, diarrhea or incontinence (Roe et al., 2002), even when a non-anticholinergic suitable alternative is available (Carnahan et al., 2004). Several investigations suggest the existence of a vicious cycle of prescription of anticholinergics and procholinergics. In fact, Gadzhanova et al. (2015) described that around 1/3 of the patients who receive AchI or memantine have received anticholinergic drugs in the previous months. They also found that $12 \%$ of de novo prescriptions of these drugs were done after the start of anti-dementia drugs. Different "combined use" prevalence rates have been found depending on the detection tool, the clinical context and the baseline disorder. For example, prevalence varies from $44.9 \%$ (with $11.3 \%$ taking a high anticholinergic load) (Cross et al., 2016), or $54.1 \%$ in hospitalized elderly (Weichert et al., 2018), to $81.3 \%$ in patients with Parkinson dementia (with 44.5\% taking a high anticholinergic load) (Mantri et al., 2019). 


\section{MEASUREMENT OF ANTICHOLINERGIC ACTION}

Anticholinergic action cannot be directly measured. Traditionally, anticholinergic load has been inferred through the use of laboratory techniques or scales and lists of anticholinergic drugs.

\section{Lab Techniques}

The serum anticholinergic activity (SAA) is the most frequently used laboratory technique and has been considered the gold standard method for measuring anticholinergic action for years (Tune and Coyle, 1980). It measures the peripheral anticholinergic action, thus not reflecting the central action. Under baseline conditions no anticholinergic activity is detected in serum, although it might be detected in the absence of drugs with anticholinergic action, due to a non-specific reaction to stress or the use of polypharmacy (Flacker and Lipsitz, 1999; Plaschke et al., 2010). In addition, it does not differentiate the agonistic or antagonistic activity of the muscarinic receptor (Collamati et al., 2016) and it does not assess the effects of metabolites such as $\mathrm{N}$-desmethylclozapine. The combination of anticholinergic drugs of different potency can increase or decrease the anticholinergic action of the drug with greater anticholinergic action, which could also happen if an anticholinergic drug is combined with a pro-cholinergic drug (Mayer et al., 2016). Other limitations to its clinical use are cost and availability.

Alternative laboratory techniques are being studied, such as blood acetylcholinesterase and blood butyrylcholinesterase levels. These techniques have shown high sensitivity in detecting drugs with anticholinergic action, but not in assessing synergistic effects of different anticholinergic agents (Plaschke et al., 2016).

\section{Scales and Indexes of Anticholinergic Drugs}

Drug scales are objective, reproducible and easy-to-administer tools to determine anticholinergic action. They can be applied in a short time, especially when online calculators are used. Many of them are based on SAA published data, so they present the limitations already described. In addition, they have other limitations such as not considering pharmacological interactions, not being homogeneous in terms of anticholinergic load attribution (e.g., in the case of quetiapine) (Brombo et al., 2018), and not usually considering the daily dose, receptor affinity, blood-brain barrier permeability, serum or tissue concentrations (Ruxton et al., 2015), renal and hepatic functions, comorbidities, and the use of other drugs. As additional limitations, they may also be outdated, and thus, underestimate the anticholinergic load, or be ambiguous because they do not indicate if there is a lack of AA or simply this has not been assessed (Bishara et al., 2017). The obtained results are not completely translatable worldwide, since these scales may include drugs that are not commercialized or approved in a specific country. In addition to all these limitations, it must be pointed out that these scales tend to assume that the anticholinergic load is linear and additive when it is unlikely that it is proportional to a ratio of $0: 1: 2: 3$ (Salahudeen et al., 2015). Furthermore, considering the finite number of muscarinic receptors, a plateau effect can be reached at some point, which in fact implies that the sum of the anticholinergic effects of the individual drugs may overestimate the total anticholinergic load. Despite all these limitations, scales seem more useful than in vitro determinations to facilitate clinical decisions (Wawruch et al., 2012).

The scales more frequently used are the following:

- Anticholinergic Drug Scale (ADS). It classifies drugs according to their AA. Scores varies from 0 , for lack of activity; 1, if a possible anticholinergic action exists, to 2-3 for high activity. The SAA, the pharmacological characteristics of each drug and the clinical experience were considered for its design. It includes a large number of medications, including non-oral formulations (Carnahan et al., 2006).

- Anticholinergic Risk Scale (ARS). Its scoring system is similar to the ADS scale, ranging from 0 to 3 points. It is based on the dissociation constant of the muscarinic receptor, the rates of anticholinergic effects and a literature review of about 500 drugs. It includes a smaller number of drugs, mainly psychotropics, and excludes non-oral formulations (Rudolph et al., 2008).

- Anticholinergic Cognitive Burden Scale (ACB). In this scale, drugs are classified according to SAA or in vitro affinity to muscarinic receptors. Drugs with ability to produce SAA or to bind muscarinic receptors in vitro score 1 , even if no relevant cognitive effects are shown. Drugs score 2 when some cognitive effects can be found and 3 when these cognitive effects are very significant. This scale includes a number of medicines similar to the ADS scale and is the scale most commonly used in research studies (Boustani et al., 2008).

- Drug Burden Index (DBI). It is not a scale, but a summation of the anticholinergic load, the sedative load or the combination of the anticholinergic load and the sedative load. This index is calculated by the ratio between the prescribed dose and the sum of the minimum dose and the prescribed dose. The greater the difference between the minimum dose and the prescribed dose, the greater the individual load for that drug (Hilmer et al., 2007). Its values range from 0 to $\infty$. Besides oral formulations, it also includes topical or inhaled drugs. As an advantage, it considers the drug dose, although it does not differentiate between mild or potent anticholinergics (Wouters et al., 2017). In a systematic review it was considered the best tool for the longitudinal evaluation of anticholinergic effects (Cardwell et al., 2015).

- Other tools used to measure anticholinergic action include the Han et al. (2001), Chew et al. (2008), and Durán et al. (2013) lists, as well as the following scales: Anticholinergic Burden Classification Scale (ABC) (Ancelin et al., 2006), Anticholinergic Activity Scale (AAS) (Ehrt et al., 2010), Anticholinergic Loading Scale 
(ACL) (Sittironnarit et al., 2011) and Anticholinergic Impregnation Scale (AIS) (Briet et al., 2017).

These different scales have been compared in several studies. One study showed that only 29 drugs were present in all the main scales used (ACB, ARS, and ADS) and only 20 if DBI was included (Naples et al., 2015). These scales included a wide number of drugs, and the correlations among them are usually low. Therefore, results among scales are different and not interchangeable (Brombo et al., 2018). ADS and ACB have an adequate correlation as their design is based on similar data, and are the scales that detect more anticholinergic drugs (Pont et al., 2015). It has been suggested that the ARS scale is better for the detection of cognitive impairment than the ACB scale, whereas the ACB is better to assess functionality, although it has not been clearly established that some tools are more specific than others for assessing specific side effects (Pasina et al., 2013).

In summary, there are several techniques to indirectly assess anticholinergic activity of drugs. The gold standard is the determination of SAA. However, this technique is not usually accessible and has some limitations. Several rating scales for anticholinergic drugs have been developed, a procedure that is more accessible, but also limited. The correlation between these scales is not high and there is little evidence of superiority of one over the other either in general or to detect specific outcomes.

\section{OUTCOMES}

Different outcomes have been associated to the intake of anticholinergic drugs, beyond cognitive disturbance. However, when studying this topic, it is important to consider reversecausation bias, i.e., that the medical condition itself, and not the anticholinergic drug used to treat it, causes the outcome. This limitation is normally solved in longitudinal studies. However, longitudinal studies are scarce since they are difficult and expensive to perform. Another difficulty is that drugs with anticholinergic action may bind to different receptors other than the muscarinic receptor which may increase or avoid certain side effects related to the anticholinergic effect (Ruxton et al., 2015).

\section{Cognitive Outcomes in Healthy Subjects}

Adverse cognitive effects of anticholinergic drugs have been evaluated in cross-sectional and longitudinal studies in healthy subjects, in subjects with MCI and in subjects with dementia. However, there is no unanimous evidence of the cognitive effects of anticholinergic drugs and whether these effects are reversible or not (Salahudeen and Nishtala, 2016). The studies in which no association was found have been questioned by a possible protopathic bias or reverse causation due to the greater use of these drugs to treat dementia prodrome symptoms. Another artifact could be the use of the Mini-Mental State Examination (MMSE), the most widely used cognitive screening test, which is now being questioned as an adequate tool to detect subtle changes induced by drugs (Salahudeen et al., 2016). In this line, more extensive neuropsychological batteries are now recommended instead (Wouters et al.,
2017). Regarding the heterogeneity of the results, another hypothesis suggests that it may be due to the heterogeneity of the cholinergic reserves of the participants (Collamati et al., 2016) which could explain the lack of association between cognition and anticholinergic load in middle-aged persons (Limback-Stokin et al., 2018).

Already in 1973 it was suggested that blocking muscarinic receptors altered learning processes (Crow and GroveWhite, 1973). Subsequent cross-sectional studies supported an association between anticholinergic load and different cognitive parameters, such as episodic memory, executive functions and psychomotor speed (Ziad et al., 2018), recall of a short story and a list of words (Potamianos and Kellett, 1982), visual immediate memory and verbal fluency (LechevallierMichel et al., 2005), immediate memory and delayed memory (Nakra et al., 1992), memory and registry domains of the MMSE (Konishi et al., 2010), color and interference in the Stroop test (Sittironnarit et al., 2011) and free immediate memory, total immediate memory, free delayed memory and total delayed memory (Fortin et al., 2011).

Despite the importance of cross-sectional studies, it has been suggested that for some cognitive domains, dysfunction could only be proved in longitudinal studies (Gnjidic et al., 2012). In addition, these studies can assess if anticholinergic drugs increase the risk of dementia or produce a reversible cognitive dysfunction. It has also been suggested that even an initially asymptomatic anticholinergic exposure can cause cognitive dysfunction in the long term after a prolonged used of anticholinergic drugs (Han et al., 2008). In longitudinal studies, anticholinergic action can accelerate the decline of the components of executive function such as attention, sequencing, concentration and cognitive flexibility (Bottiggi et al., 2006).

Intake of anticholinergics has been associated with a greater decline of MMSE scores (Fox et al., 2011b) regardless of functional decline (Brombo et al., 2018). A brief exposure to drugs with anticholinergic action is associated with cognitive impairment, but not with dementia(Ancelin et al., 2006; Jamsen et al., 2017). In contrast, a prolonged exposure has been associated with dementia and not only with cognitive impairment (Cai et al., 2013). The association between long-term exposure and dementia suggests that the time factor and/or the cumulative dose influence the development of a neurodegenerative process. However, it is currently unknown whether the time exposure factor is a necessary or sufficient condition to increase the risk of dementia. Thus, Whalley et al. (2012) showed that previous cognitive impairment is more frequent in patients who took anticholinergics.

In relation to the transition from cognitive normality to MCI, Campbell et al. (2018) suggested that the continued use of potent anticholinergics may increase conversion rates. In contrast, Chuang et al. (2017) argued that the continued use and not the high anticholinergic load is related to incident MCI and dementia, as high potency anticholinergics are usually prescribed only for short periods of time. In this line, other works have also found a higher risk of incident dementia (Carrière et al., 2009), finding even a dose-dependent relationship (Jessen et al., 2010; Gray et al., 
2015; Richardson et al., 2018). Some authors have suggested that the exposure to potent anticholinergics and the risk of dementia or Alzheimer's disease is independent of the time elapsed from such exposure to the moment of diagnosis (Gray et al., 2015). Nevertheless, the relationship between the intermittent use of anticholinergic drugs and cognitive effects in the medium and long term is not yet sufficiently clear (Araklitis et al., 2017).

The prolonged use of medications with anticholinergic activity such as antidepressants, urological and antiparkinsonian drugs has been associated with increased risk of dementia (Richardson et al., 2018). In the case of antidepressants, reverse causation is suspected if the drug has not been used for a long time.

The presence of the APOE 4 allele is widely recognized as a risk factor of dementia. However, the results about the influence of APOE 4 allele on cognitive dysfunction mediated by anticholinergic drugs are contradictory. While cross-sectional studies support E4 allele to be associated with poorer cognitive performance (Pomara et al., 2004; Uusvaara et al., 2009), longitudinal studies either found no association (Gray et al., 2015) or deterioration in MMSE scores only in females (Carrière et al., 2009).

Summing-up the information related to cognitively normal persons, the self-limited anticholinergic action on cognition in this population is well known long ago. It requires the interaction between the previous cognitive level and the potency of the anticholinergic action. However, while some longitudinal studies have found a relationship between the continued use of anticholinergic drugs and the development of Alzheimer's pathophysiology, others have only found a reversible cognitive impairment. Therefore, it is suggested that the exposure time or the cumulative dose of anticholinergics may end up exceeding a threshold from which the neurodegenerative process is triggered and the anticholinergic effect is not reversible anymore.

\section{Cognitive Outcomes in Previously Cognitive Impaired Patients}

The anticholinergic load may be a modifiable risk factor for dementia (Green et al., 2016). It is estimated that between 2 and $12 \%$ of patients with suspected dementia actually have pharmacological iatrogenesis in that respect (Moore and O'Keeffe, 1999). Patients with SAA levels at a 90th percentile or higher were 13 times more likely to have a MMSE of 24 or lower compared to patients without detectable SAA (Mulsant et al., 2003). In addition, a greater progression to MCI or dementia has also been found if both anticholinergic load and positivity to beta-amyloid are present (Risacher et al., 2016).

In a sample of primary care patients with first cognitive complaints, an elevated anticholinergic load was required for a significant association with cognitive dysfunction to be found (Grande et al., 2017). In contrast, in patients with questionable cognitive impairment (QCI), cognitive function improved when they received anticholinergics, due to the therapeutic effects on comorbid pathologies (Swami et al., 2016). According to this, Fox et al. (2011a) have suggested that the anticholinergic action has to reach a critical threshold to produce cognitive effects. In addition, they postulate that there is a floor effect in the most advanced stages of neurodeterioration, in which cognition is not altered. The same group showed that the effect is higher in MMSE scores above 26 and is non-existent in scores below 21 (Fox et al., 2011b).

The combined use of anticholinergic drugs and AchI produces a higher rate of cognitive (Lu and Tune, 2003) and functional decline (Sink et al., 2008) with respect to those not taking anticholinergics at the same time, and this may induce an early discontinuation of the AchI treatment because its beneficial effect is being blocked by the anticholinergic drug due (Sverdrup Efjestad et al., 2017). However, higher cognitive decline of the combined treatment has been questioned in other studies (Bottiggi et al., 2007).

Despite the drawbacks of taking anticholinergic drugs, a novel approach that takes advantage of the peripheral anticholinergic action has been proposed in Alzheimer's disease. A single-blind study with 41 moderate probable Alzheimer's disease subjects showed that, after the coadministration of donepezil with the peripheral anticholinergic drug solifenacin $15 \mathrm{mg}$ /day allowed the titration of donepezil up to $38 \mathrm{mg} /$ day without notable adverse effects in $88 \%$ of patients. At 26 weeks, co-administration of solifenacin significantly improved the score of the ADAS-Cog, a widely used cognitive scale, versus baseline and versus the standard treatment with donepezil $10 \mathrm{mg} /$ day. These results suggest that concomitant administration of AchI and a peripherally acting anticholinergic drug increases efficacy and tolerability of Alzheimer's disease treatments (Chase et al., 2017). Nevertheless, the question remains on whether the addition of an AchI to a long-term treatment with anticholinergic drugs with central action that are not susceptible to modification could reduce the deleterious effects on cognition, functionality or mortality.

In brief, the higher the cognitive impairment the patients has, the more damaging is the effect of the anticolinergic effects of drugs, although this deleterious cognitive action may require exceeding a threshold to manifest. However in patients with advanced cognitive impairment, a ground effect may be found, and they will not present greater cognitive impairment when a certain level of deterioration is reached.

\section{Cognitive Outcomes in Schizophrenia}

Cholinergic function in relation to cognition in schizophrenia has been recently studied in more detail. Patients with schizophrenia have twice the risk of developing dementia before the age of 80 than the general population (Tsoutsoulas et al., 2017). Cholinergic and dopaminergic systems, and the balance between them are essential for cognition, especially in people with schizophrenia (Ang et al., 2017). Unlike in affective disorders, in schizophrenia there is a low brain density of muscarinic receptors and patients usually have early cognitive dysfunction (Tsoutsoulas et al., 2017). Therefore, a light anticholinergic load can have a significant cognitive impact in schizophrenia, a finding not observed in schizoaffective disorder or bipolar disorder patients (Eum et al., 2017). In this line, data points toward a selective action of the M1 receptor in memory processes in schizophrenia. Thus, 
highly selective agonists of the M1 receptor would obtain better cognitive results than AchI, which are less specific (Vingerhoets et al., 2017).

Neuroimaging studies in patients with schizophrenia have found higher SAA related to the use of medications. Subjects with higher SAA have lower brain activation in the dorsolateral, anterior and medial prefrontal areas. These studies hypothesize that SAA interrupts modulatory prefrontal cholinergic transmission (Schreiber et al., 2018).

Ang et al. (2017) suggested that while cognitive impairment by anticholinergics in schizophrenia exists, it has a low effect size. A different study demonstrated a worse performance in speed processing and verbal memory related to the dose of antipsychotics and not to their anticholinergic action (Rehse et al., 2016). They also found that the worst performance was encountered if the antipsychotic drug had also a high anticholinergic action, except in patients receiving clozapine, a drug with clear anticholinergic effect. Considering these results, it is possible to hypothesize that NMDA agonism and M4 agonism can compensate for the anticholinergic action.

In patients with schizophrenia older than 50 years, a high anticholinergic load is associated with problems in spatial working and immediate memory and visuospatial ability. Deficits in attention, executive function and processing speed in schizophrenia patients may favor the presentation of a floor effect that masks the anticholinergic action. Anticholinergics may contribute to specific cognitive deficits in elders with schizophrenia that resemble those present in Alzheimer's disease (Tsoutsoulas et al., 2017). On the other hand, there is strong evidence that if anticholinergic load is reduced in patients with schizophrenia, the performance of verbal memory and attention improves (Rehse et al., 2016).

At a functional level, the anticholinergic load associated with psychotropic drugs can alter the ability to participate and benefit from psychosocial treatments (O'Reilly et al., 2016).

In general, anticholinergic action of drugs in patients with schizophrenia are associated to worse cognitive outcomes. In fact, cognitive outcomes improve as the anticholinergic burden is reduced. However, it is suggested that other neurotransmitter systems may be involved in such cognitive impairment, since Clozapine shows better cognitive effects than others despite its intense anticholinergic action.

\section{Delirium}

Delirium is a highly prevalent process in the elderly, especially in those suffering from dementia. Its onset has frequently been associated with anticholinergic drugs treatment (Collamati et al., 2016). The most accepted theory regarding delirium is the presence of diffuse imbalance of cerebral neurotransmission that implicates muscarinic receptors among others (Collamati et al., 2016). There is a biological plausibility about the association between anticholinergic drugs and delirium, but to date there are no conclusive results (Campbell et al., 2011; Wolters et al., 2015; Moorey et al., 2016). The variability of the scales used, age, presence of dementia, medical comorbidity, nutritional status and the possibility of reverse causation are among the most cited confounding factors (Collamati et al., 2016; Egberts et al., 2017).

\section{Emergence of Psychiatric Symptoms}

Anticholinergic action has been related to the onset of psychiatric symptoms (mainly in the psychotic sphere) in elderly population with and without dementia, although the number of studies is still reduced.

In addition, it has been ruled out that this disposition to psychosis occurs indirectly due to cognitive function worsening (Cancelli et al., 2008). On the contrary, the procholinergic action of the AchI may be effective for controlling psychotic symptoms in dementia.

In a study in Alzheimer's disease, the intake of anticholinergic drugs (except antipsychotics, to avoid reverse causation) increased the risk of psychosis, showing more risk for an increased anticholinergic load (Cancelli et al., 2008). This is in line with another study where the presence of SAA was associated with delusional ideation and diurnal rhythm disturbances (Hori et al., 2011). However, a different study (Dauphinot et al., 2017) found that the initial relationship between the anticholinergic load and the higher score in the Neuropsychiatric Inventory (NPI), a widely used scale to measure mental symptoms in neurodegenerative diseases, became nonsignificant after adjusting for confounding factors.

Hori et al. (2011) have also commented on the existence of the aforementioned "vicious cycle of anticholinergic activity in Alzheimer's disease," in which polypharmacy or the use of potent anticholinergics generates SAA and, in turn, this AA produces psychiatric symptoms that will be addressed with the prescription of new psychotropics with anticholinergic action.

In the face of risk factors such as old age, dementia, brain lesions, psychotic depression or bipolar disorder, cases of complex visual hallucinations related to the combination of serotonergic hyperactivity and cholinergic hypoactivity have been described. However, this a situation that is more frequent in patients on antidepressants, especially tricyclics (Cancelli et al., 2004).

\section{Functional Outcomes}

Several studies have tried to correlate anticholinergic drugs with physical and functional deterioration. Treatmentrelated alterations would be due to their central action, but also to peripheral effects such as the difficulty with visual accommodation, tachycardia or gait problems.

The large majority of cross-sectional and longitudinal studies reviewed associate anticholinergic load measured with different instruments and loss of functionality. However, there are some studies that do not find a relationship between anticholinergic load and loss of functionality (Bostock et al., 2013; Sanders et al., 2017).

Mayer et al. (2017) found that the ARS and ADS scales had a high association with the Barthel index, a well-known scale to measure functionality. In a systematic review Wouters et al. (2017) concluded that the DBI was associated with changes in gait and basic and instrumental activities of daily life. The basic activities most affected by the anticholinergic action were bathing, grooming, dressing, transfers, mobility and stairs use (Lowry et al., 2011). A progressive decrease in global activity measured by accelerometers was also found (Clarke et al., 
2018). A dose-response relationship between anticholinergic load and dysfunction has also been described. In fact, higher doses have a greater effect on people with more preserved previous functioning, and there is a floor effect in the most deteriorated patients (Sink et al., 2008).

The anticholinergic action affects the capacity for functional rehabilitation of both severe processes such as a stroke (Kose et al., 2018) and apparently minor injuries such as an ankle sprain (Kröger et al., 2019).

There are scarce studies regarding the influence of anticholinergic treatment on quality of life and they report contradictory data (Cossette et al., 2017; Wouters et al., 2017). Generally, there seems to be less psychological well-being in the patients taking anticholinergics, although it is not possible to exclude reverse causation because this association is not maintained when excluding patients diagnosed with depression (Teramura-Grönblad et al., 2011).

With regard to falls, it is estimated that up to $40 \%$ of community-dwelling elderly and even more of those living in institutions experience falls annually, with serious injury or mortality in $10 \%$ of cases (Marcum et al., 2015). In fact, falls are a frequent cause of hospitalization and institutionalization among old people (Zia et al., 2016). Anticholinergics intake has been associated with the presence of recurrent falls, although there is no definitive evidence in this regard (Lattanzio et al., 2018). It has been suggested that drugs with anticholinergic action may not be independently associated with the risk of falls and fractures (Fraser et al., 2014). The association between falls and anticholinergics could be related to dosage or duration of treatment, or to their non-anticholinergic effects such as sedation or orthostasis (Marcum et al., 2015), and mediated by the presence of changes in gait and balance (Zia et al., 2016). In fact, Wilson et al. (2011) found that only SSRI antidepressants, and no other anticholinergic drugs, have been associated with an increased risk of falls.

Thus, studies on the functional effects of anticholinergics have not shown unanimous results, but the large majority of them suggest a relationship between anticholinergic load and loss of functionality, with a threshold and a ground effect to produce these effects. With respect to falls, there seems to be a joint presentation of taking drugs with anticholinergic action and falls, although these falls could also be related to other patients' factors and characteristics of drugs and not directly with anticholinergic action.

\section{Hospitalization and Outpatient Care}

A systematic review found a relationship between anticholinergic load and hospitalization in five of the six included papers (Wouters et al., 2017). In institutionalized elderly, a moderate anticholinergic load increased the risk of hospitalization (Vetrano et al., 2016). A common finding is that hospitalization increases the percentage of patients who take anticholinergics, the number of anticholinergics per patient and the total anticholinergic load with respect to non-hospitalized patients due to reasons associated to hospitalization and/or treatment needs (Collamati et al., 2016). Additionally, a cohort study found an association between anticholinergic load and duration of hospital admission in both, patients with and without dementia (Gnjidic et al., 2014); however, this association was not found in other studies (Lowry et al., 2011; Egberts et al., 2017).

A higher risk of institutionalization in nursing homes has been found to be proportional to the anticholinergic load regardless of the presence of delirium and after adjustment for comorbid conditions (Egberts et al., 2017). In addition, a greater anticholinergic load has been associated with a greater number of outpatient visits and visits to the emergency room (Campbell et al., 2016).

So, in general, both institutionalization and hospitalization appear temporarily associated with the use of anticholinergic drugs. However, the relationship between hospitalization and its characteristics and the consumption of anticholinergic drugs before and after hospitalization could be indirect and due to one of the other multiple factors involved.

\section{Mortality}

Cardiovascular anticholinergic effects such as tachyarrhythmia, syncope, ischemia or neurological effects such as epileptic seizures, hallucinations, and delirium may increase mortality (Vetrano et al., 2016; Wouters et al., 2017).

There are no conclusive results about greater mortality in hospitalized elderly patients taking anticholinergics. However, mortality has been associated with anticholinergic use in the presence of hyponatremia (Lowry et al., 2011) or delirium (Mangoni et al., 2013), especially in the presence of cognitive impairment (Lattanzio et al., 2018), even 1 year after discharge.

Anticholinergics have also been found to be associated with higher mortality in institutionalized elderly, especially in those with coronary disease (Vetrano et al., 2016) due to the greater vulnerability to the proarrhythmic effects of these drugs. A longitudinal study found a dose-dependent association between taking anticholinergics and the risk of stroke and death in elderly people with Alzheimer's disease, mixed dementia and vascular dementia (Tan et al., 2018) which would parallel to the well-known risk of antipsychotics. Nevertheless, this risk does not seem to be directly associated to the possible anticholinergic effect of antipsychotics, since it is very different among the different drugs (Maust et al., 2015).

\section{CONSEQUENCES OF DISCONTINUATION OF ANTICHOLINERGIC DRUGS}

Deprescription has the potential to reduce or eliminate unnecessary or potentially inadequate drugs, reduce polypharmacy and improve different health variables. Eliminating or reducing the anticholinergic load can improve cognition, adverse effects and quality of life (Lupu et al., 2017). For example, in patients with schizophrenia younger than 50 years, the discontinuation of anticholinergics improved the results in the Wisconsin Card Sorting Test, a measurement of executive dysfunction (Sathienluckana et al., 2018).

However, some experts suggest that there is little evidence regarding the reversibility of anticholinergic cognitive effects 
through discontinuation and question the safety of this measure. Different studies have proposed conflicting hypotheses regarding the reversibility of cognitive effects after discontinuation. Several of them point to a long-term irreversibility, although it is unknown whether a temporary threshold for such irreversibility exists. In a systematic review including four studies (two randomized controlled trials and two prospective cohort studies), only the cohort studies showed cognitive improvement after anticholinergic drugs discontinuation (Salahudeen et al., 2014). In contrast, a different prospective cohort study conducted by Gray et al. (2015) did not find attenuation of the risk of dementia or Alzheimer's disease with discontinuation. A subanalysis of a single-blind randomized trial that did not find cognitive improvement showed that the subgroup of patients in whom the anticholinergic load could be eliminated had a greater improvement in immediate memory. This finding suggests that the cognitive improvement could be dose- dependent or could require the total discontinuation of anticholinergics (Kersten et al., 2013). At the same time, it has also been suggested that a long anticholinergic-free period is required for cognitive improvement.

Nonetheless, discontinuation is not always possible and clinical reasons for not carrying out the discontinuation should always be considered (Lupu et al., 2017). For example, in some patients with schizophrenia, withdrawing benztropine may clearly worsen extrapyramidal symptoms related to antipsychotic medication, and therefore maintenance of the treatment may be required.

Thus, the discontinuation of anticholinergic drugs should be an objective due to their adverse effects. However, this objective should be flexible because the cognitive effects may no longer be reversible or there is an evident risk of serious worsening of an underlying disease.

\section{DISCUSSION AND RECOMMENDATIONS FOR CLINICAL PRACTICE}

Geriatric prescription of anticholinergic drugs cannot always be avoided in clinical practice. If prescription is necessary, priority should be given to drugs with a mild anticholinergic load (Bishara et al., 2017) and those with high affinity for their site of action (Collamati et al., 2016). In addition, possible medication management problems should be addressed in view of the increased anticholinergic load. In fact, this has been related to adherence problems especially in male patients (Boccardi et al., 2017).

When an anticholinergic drug is prescribed, cognitive function and comorbidities sensitive to anticholinergic effects such as benign prostatic hypertrophy, constipation, gait problems, among others, should be assessed, together with the already existing avoidable or inevitable anticholinergic load caused by concomitant treatments.

Given the potential risk of irreversible cognitive effects in prolonged treatments, the efficacy and risks of anticholinergic drugs should be re-evaluated when treatment periods longer than 3 months are considered (Cai et al., 2013; Wu et al., 2017).
Abrupt discontinuation is not recommended due to the risk of a cholinergic rebound, i.e., symptoms of agitation, diarrhea, vomiting, lacrimation, tachycardia, insomnia and movement disorders after withdrawal (Lupu et al., 2017). Patients should be informed of potential risks of discontinuation, and participate in therapeutic decisions (Bishara et al., 2017).

Iatrogenesis caused by anticholinergic drugs could be reduced with the availability of the opinion of a clinical pharmacologist, the use of anticholinergic load calculators, tools such as the "Pocket Reference Card for Medicines with Anticholinergic Activity" or future software that generates therapeutic alternatives to specific clinical problems (Salahudeen and Nishtala, 2016).

The following are practical recommendations oriented to different clinical conditions.

- 'Dementias, including neuropsychiatric symptoms in dementia.' The anticholinergic risk is higher in dementias with cholinergic deficit such as Alzheimer's disease, Lewy body dementia and Parkinson's dementia. When the use of AchI produces procholinergic adverse effects, low doses should be used, titration should be slower, or their use should be abandoned, instead of prescribing anticholinergic drugs to alleviate these side effects. In a patient presenting symptoms of dementia, a discontinuation of anticholinergics that results in cognitive improvement should question the neurodegenerative origin of the problem, or at least consider that the medication has acted as an AA insert, which means that the cholinergic system is altered but is functional without external interference. On the contrary, the absence of improvement after the discontinuation of the anticholinergic drug would favor the diagnosis of dementia. If the use of antipsychotics is necessary, those with less anticholinergic action should be prioritized. In fact, in Europe, Risperidone is the only atypical antipsychotic licensed drug for the treatment of neuropsychiatric symptoms in dementia. The anticholinergic burden attributed to Risperidone varies in the different medication lists. There are other antipsychotic drugs, such as Aripiprazole, considered less anticholinergic. However, nowadays the main focus on the safety of the use of antipsychotics in dementia is oriented to the greater risks of cerebrovascular events and death. These effects are serious, and the reason why antipsychotics in dementia should only be prescribed in the short term and only after having tried non-pharmacological options.

- Schizophrenia. Antipsychotics and anticholinergic correctors used for extrapyramidal side effects of antipsychotics are negatively associated with cognition (Ziad et al., 2018). The cognitive effects of anticholinergics should be closely monitored in patients with greater baseline cognitive impairment. Except in the acute phases, the minimum doses of antipsychotic drugs should be used to avoid or reduce the additional use of anticholinergic correctors for extrapyramidal side effects, which must be avoided as much as possible in the elderly 
(Rehse et al., 2016; Lupu et al., 2017). This should be considered in all cases but in acute or tardive dystonias that respond to anticholinergics the withdrawal may not be possible (Lupu et al., 2017). Since schizophrenia is a chronic disease, the anticholinergic load in patients suffering from it can increase with treatment time and by the treatments prescribed for other comorbidities (Tsoutsoulas et al., 2017).

- Acute hallucinatory episode. The action of antidepressants and other serotonergic and/or anticholinergic drugs should be assessed in order to withdraw them before considering the prescription of antipsychotics (Cancelli et al., 2004).

- Depression. Given the risk of progression to dementia in an insufficiently treated depression, the first measure is to try to achieve remission. In a limited number of cases, patients with prolonged treatment with tricyclic antidepressants could be harmed by switching to SSRIs if their effectiveness on depression is lower (Lupu et al., 2017). Among antidepressants, amitriptyline has a high anticholinergic load already at a dose of $25 \mathrm{mg} /$ day, nortriptyline has a moderate load at $50 \mathrm{mg} /$ day and paroxetine has also a moderate load at $30 \mathrm{mg} /$ day (Chew et al., 2008).

- Anxiety. Benzodiazepines may cause cognitive impairment due to several different mechanisms, including anticholinergic effects when prescribed at high doses. Lorazepam has a short action and is low fat-soluble, causing less cognitive effects than other benzodiazepines (Bishara et al., 2017).

- Parkinson's disease. Anticholinergic drugs should be discontinued if cognitive dysfunction occurs and should no longer be reintroduced (Nishiyama et al., 1998).

- Cardiovascular diseases. Drugs with anticholinergic action such as warfarin or digoxin are essential for the prevention of cardiovascular and cerebrovascular events and do not always have adequate alternatives (Pasina et al., 2019). Therefore, their use should be prioritized over other drugs with anticholinergic action that may be necessary to prescribe. Additionally, some drugs with cardiovascular action can exert compensatory protective effects on cognitive dysfunction (Richardson et al., 2018).

- Urinary incontinence/bladder dysfunction. Nonpharmacological alternatives such as changes in lifestyle, bladder retraining and physiotherapy should be considered first (Araklitis et al., 2017). If anticholinergics are prescribed and clearly improve symptoms and quality of life, discontinuation may not be appropriate. Among the

\section{REFERENCES}

American Geriatrics Society 2012 Beers Criteria Update Expert Panel (2012). American Geriatrics society updated beers criteria for potentially inappropriate medication use in older adults. J. Am. Geriatr. Soc. 60, 616-631. doi: 10.1111/j. 1532-5415.2012.03923.x

Ancelin, M. L., Artero, S., Portet, F., Dupuy, A. M., Touchon, J., and Ritchie, K. (2006). Non-degenerative mild cognitive impairment in elderly people and use of anticholinergic drugs: longitudinal cohort study. BMJ 332, 455-459. doi: 10.1136/bmj.38740.439664.de drugs of its class, tolterodine and oxybutynin are the urinary incontinence drugs with the highest anticholinergic load (Araklitis et al., 2017). Oxybutynin has the highest blood-brain barrier permeability and trospium the lowest. Darifenacin has the lowest risk of interaction with AchI (Sverdrup Efjestad et al., 2017).In multiple sclerosis, alternatives such as the use of botulinum toxin injections or mirabegron should be considered (Morrow et al., 2018).

\section{CONCLUSION}

Anticholinergic agents are clearly useful for the treatment of several processes that involve different organs and systems, such as cardiovascular, respiratory and CNS s, among others. In the elderly, side effects are more commonly found. They can manifest acutely, such as constipation, reversible cognitive dysfunction or delirium, or after a long exposure, such as an increased risk of developing dementia, hospitalization or increased mortality.

The risk-benefit ratio should always be assessed when prescribing a drug with anticholinergic effects. It is important to balance the risk of using them and the risk of not using them and to evaluate pharmacological and nonpharmacological alternatives. Assessment must always include medical comorbidities and other medications that the patient is receiving. To sum up, the research line that differentiates adequately and inadequately prescribed anticholinergic drugs seems very promising and more studies should be done considering the knowledge gaps reviewed.

\section{AUTHOR CONTRIBUTIONS}

JL-Á and LA-O designed the project. JL-Á managed the literature search and wrote the first draft of the manuscript. LA-O and JS-L-J revised the drafts and supervised the English translation. JL-Á, LA-O, and JS-L-J reviewed and contributed equally to the final version of the manuscript.

\section{FUNDING}

Open access publication fees have been supported by the Departamento de Medicina Legal, Psiquiatría y Patología, Facultad de Medicina, Universidad Complutense de Madrid, Madrid, Spain.
Ang, M. S., Abdul Rashid, N. A., Lam, M., Rapisarda, A., Kraus, M., Keefe, R. S. E., et al. (2017). The impact of medication anticholinergic burden on cognitive performance in people with Schizophrenia. J. Clin. Psycho. Pharmacol. 37, 651-656. doi: 10.1097/JCP.0000000000000790

Araklitis, G., Thiagamoorthy, G., Hunter, J., Rantell, A., Robinson, D., and Cardozo, L. (2017). Anticholinergic prescription: are healthcare professionals the real burden? Int. Urogynecol. J. 28, 1249-1256. doi: 10.1007/s00192-0163258-3

Bala, S. S., Jamieson, H. A., and Nishtala, P. S. (2019). Determinants of prescribing potentially inappropriate medications in a nationwide cohort of community 
dwellers with dementia receiving a comprehensive geriatric assessment. Int. J. Geriatr. Psychiatry. 34, 153-161. doi: 10.1002/gps.5004

Bishara, D., Harwood, D., Sauer, J., and Taylor, D. M. (2017). Anticholinergic effect on cognition (AEC) of drugs commonly used in older people. Int. J. Geriatr. Psychiatry 32, 650-656. doi: 10.1002/gps.4507

Boccardi, V., Baroni, M., Paolacci, L., Ercolani, S., Longo, A., Giordano, M., et al. (2017). Anticholinergic burden and functional status in older people with cognitive impairment: results from the regal project. J. Nutr. Health Aging 21, 389-396. doi: 10.1007/s12603-016-0787-x

Bostock, C. V., Soiza, R. L., and Mangoni, A. A. (2013). Associations between different measures of anticholinergic drug exposure and Barthel Index in older hospitalized patients. Ther. Adv. Drug Saf. 4, 235-245. doi: 10.1177/ 2042098613500689

Bottiggi, K. A., Salazar, J. C., Yu, L., Caban-Holt, A. M., Ryan, M., Mendiondo, M. S., et al. (2006). Long-term cognitive impact of anticholinergic medications in older adults. Am. J. Geriatr. Psychiatry 14, 980-984. doi: 10.1097/01.jgp. 0000224619.87681 .71

Bottiggi, K. A., Salazar, J. C., Yu, L., Caban-Holt, A. M., Ryan, M., and Schmitt, F. A. (2007). Concomitant use of medications with anticholinergic properties and acetylcholinesterase inhibitors: impact on cognitive and physical functioning in Alzheimer disease. Am. J. Geriatr. Psychiatry 15, 357-359. doi: 10.1097/jgp. 0b013e31802dd354

Boustani, M. A., Campbell, N. L., Munger, S., Maidment, I., and Fox, C. (2008). Impact of anticholinergics on the aging brain: a review and practical application. Aging Health 4, 311-320. doi: 10.2217/1745509x.4.3.311

Briet, J., Javelot, H., Heitzmann, E., Weiner, L., Lameira, C., D’Athis, P., et al. (2017). The anticholinergic impregnation scale: towards the elaboration of a scale adapted to prescriptions in French psychiatric settings. Therapie 72, 427-437. doi: 10.1016/j.therap.2016.12.010

Brombo, G., Bianchi, L., Maietti, E., Malacarne, F., Corsonello, A., Cherubini, A., et al. (2018). Association of anticholinergic drug burden with cognitive and functional decline over time in older inpatients: results from the CRIME project. Drugs Aging 35, 917-924. doi: 10.1007/s40266-018-0584-9

Caccamo, A., Oddo, S., Billings, L. M., Green, K. N., Martinez-Coria, H., Fisher, A., et al. (2006). M1 receptors play a central role in modulating AD-like pathology in transgenic mice. Neuron 2, 671-682. doi: 10.1016/j.neuron.2006.01.020

Cai, X., Campbell, N., Khan, B., Callahan, C., and Boustani, M. (2013). Longterm anticholinergic use and the aging brain. Alzheimers Dement. 9, 377-385. doi: 10.1016/j.jalz.2012.02.005

Campbell, N., Perkins, A., Hui, S., Khan, B., and Boustani, M. (2011). Association between prescribing of anticholinergic medications and incident delirium: a cohort study. J. Am. Geriatr. Soc. 59(Suppl. 2), S277-S281. doi: 10.1111/j.15325415.2011.03676.x

Campbell, N. L., Lane, K. A., Gao, S., Boustani, M. A., and Unverzagt, F. (2018). Anticholinergics influence transition from normal cognition to mild cognitive impairment in older adults in primary care. Pharmacotherapy 38, 511-519. doi: $10.1002 /$ phar. 2106

Campbell, N. L., Perkins, A. J., Bradt, P., Perk, S., Wielage, R. C., Boustani, M. A., et al. (2016). Association of anticholinergic burden with cognitive impairment and health care utilization among a diverse ambulatory older adult population. Pharmacotherapy 36, 1123-1131. doi: 10.1002/phar.1843

Cancelli, I., Marcon, G., and Balestrieri, M. (2004). Factors associated with complex visual hallucinations during antidepressant treatment. Hum. Psycho. Pharmacol. 19, 577-584. doi: 10.1002/hup.640

Cancelli, I., Valentinis, L., Merlino, G., Valente, M., and Gigli, G. L. (2008). Drugs with anticholinergic properties as a risk factor for psychosis in patients affected by Alzheimer's disease. Clin. Pharmacol. Ther. 84, 63-68. doi: 10.1038/s.clpt. 6100435

Cardwell, K., Hughes, C. M., and Ryan, C. (2015). The association between anticholinergic medication burden and health related outcomes in the 'Oldest Old': a systematic review of the literature. Drugs Aging 32, 835-848. doi: 10. 1007/s40266-015-0310-9

Carnahan, R. M., Lund, B. C., Perry, P. J., and Chrischilles, E. A. (2004). The concurrent use of anticholinergics and cholinesterase inhibitors: rare event or common practice? J. Am. Geriatr. Soc. 52, 2082-2087. doi: 10.1111/j.1532-5415. 2004.52563.x

Carnahan, R. M., Lund, B. C., Perry, P. J., Pollock, B. G., and Culp, K. R. (2006). The anticholinergic drug scale as a measure of drug-related anticholinergic burden: associations with serum anticholinergic activity. J. Clin. Pharmacol. 46, 1481-1486. doi: 10.1177/0091270006292126

Carrière, I., Fourrier-Reglat, A., Dartigues, J. F., Rouaud, O., Pasquier, F., Ritchie, K., et al. (2009). Drugs with anticholinergic properties, cognitive decline, and dementia in an elderly general population: the 3-city study. Arch. Intern. Med. 169, 1317-1324. doi: 10.1001/archinternmed.2009.229

Chase, T. N., Farlow, M. R., and Clarence-Smith, K. (2017). Donepezil Plus Solifenacin (CPC-201) treatment for Alzheimer's disease. Neurotherapeutics. 14, 405-416. doi: 10.1007/s13311-016-0511-x

Chew, M. L., Mulsant, B. H., Pollock, B. G., Lehman, M. E., Greenspan, A., Mahmoud, R. A., et al. (2008). Anticholinergic activity of 107 medications commonly used by older adults. J. Am. Geriatr. Soc. 56, 1333-1341. doi: 10. 1111/j.1532-5415.2008.01737.x

Chuang, Y. F., Elango, P., Gonzalez, C. E., and Thambisetty, M. (2017). Midlife anticholinergic drug use, risk of Alzheimer's disease, and brain atrophy in community-dwelling older adults. Alzheimers Dement. 3, 471-479. doi: 10. 1016/j.trci.2017.06.004

Clarke, C. L., Sniehotta, F. F., Vadiveloo, T., Donnan, P. T., and Witham, M. D. (2018). Association between objectively measured physical activity and opioid, hypnotic, or anticholinergic medication use in older people: data from the physical activity cohort scotland study. Drugs Aging 35, 835-842. doi: 10.1007/ s40266-018-0578-7

Collamati, A., Martone, A. M., Poscia, A., Brandi, V., Celi, M., Marzetti, E., et al. (2016). Anticholinergic drugs and negative outcomes in the older population: from biological plausibility to clinical evidence. Aging Clin. Exp. Res. 28, 25-35. doi: 10.1007/s40520-015-0359-7

Cossette, B., Bagna, M., Sene, M., Sirois, C., Lefebvre, G. P., Germain, O., et al. (2017). Association between anticholinergic drug use and health-related quality of life in community-dwelling older adults. Drugs Aging 34, 785-792. doi: $10.1007 / \mathrm{s} 40266-017-0486-2$

Cross, A. J., George, J., Woodward, M. C., Ames, D., Brodaty, H., Ilomäki, J., et al. (2016). Potentially Inappropriate medications and anticholinergic burden in older people attending memory clinics in Australia. Drugs Aging 33, 37-44. doi: $10.1007 / \mathrm{s} 40266-015-0332-3$

Crow, T. J., and Grove-White, I. G. (1973). An analysis of the learning deficit following hyoscine administration to man. Br. J. Pharmacol. 49, 322-327. doi: 10.1111/j.1476-5381.1973.tb08379.x

Cummings, J. L., and Back, C. (1998). The cholinergic hypothesis of neuropsychiatric symptoms in Alzheimer's disease. Am. J. Geriatr. Psychiatry 6(2 Suppl. 1), S64-S78.

Dauphinot, V., Mouchoux, C., Veillard, S., Delphin-Combe, F., and KrolakSalmon, P. (2017). Anticholinergic drugs and functional, cognitive impairment and behavioral disturbances in patients from a memory clinic with subjective cognitive decline or neurocognitive disorders. Alzheimers Res. Ther. 9:58. doi: 10.1186/s13195-017-0284-4

Durán, C. E., Azermai, M., and Vander Stichele, R. H. (2013). Systematic review of anticholinergic risk scales in older adults. Eur. J. Clin. Pharmacol. 69, 1485-1496. doi: 10.1007/s00228-013-1499-3

Egberts, A., van der Craats, S. T., van Wijk, M. D., Alkilabe, S., van den Bemt, P. M. L. A., and Mattace-Raso, F. U. S. (2017). Anticholinergic drug exposure is associated with delirium and postdischarge institutionalization in acutely ill hospitalized older patients. Pharmacol. Res. Perspect. 5:e00310. doi: 10.1002/ prp2.310

Ehrt, U., Broich, K., Larsen, J. P., Ballard, C., and Aarsland, D. (2010). Use of drugs with anticholinergic effect and impact on cognition in Parkinson's disease: a cohort study. J. Neurol. Neurosurg. Psychiatry 81, 160-165. doi: 10.1136/jnnp. 2009.186239

Eum, S., Hill, S. K., Rubin, L. H., Carnahan, R. M., Reilly, J. L., Ivleva, E. I., et al. (2017). Cognitive burden of anticholinergic medications in psychotic disorders. Schizophr. Res. 190, 129-135. doi: 10.1016/j.schres.2017.03.034

Flacker, J. M., and Lipsitz, L. A. (1999). Serum anticholinergic activity changes with acute illness in elderly medical patients. J. Gerontol. A Biol. Sci. Med. Sci. 54, M12-M16.

Fortin, M. P., Rouch, I., Dauphinot, V., Gédéon, C., Genthon, S., Bonnefoy, M., et al. (2011). Effects of anticholinergic drugs on verbal episodic memory function in the elderly: a retrospective, cross-sectional study. Drugs Aging 28, 195-204. doi: 10.2165/11586580-00000000000000 
Fox, C., Livingston, G., Maidment, I. D., Coulton, S., Smithard, D. G., Boustani, M., et al. (2011a). The impact of anticholinergic burden in Alzheimer's dementiathe LASER-AD study. Age Ageing 40, 730-735. doi: 10.1093/ageing/afr102

Fox, C., Richardson, K., Maidment, I. D., Savva, G. M., Matthews, F. E., Smithard, D., et al. (2011b). Anticholinergic medication use and cognitive impairment in the older population: the medical research council cognitive function and ageing study. J. Am. Geriatr. Soc. 59, 1477-1483. doi: 10.1111/j.1532-5415.2011. 03491.x

Fraser, L. A., Adachi, J. D., Leslie, W. D., Goltzman, D., Josse, R., Prior, J., et al. (2014). for the CaMos research group. effect of anticholinergic medications on falls, fracture risk, and bone mineral density over a 10 -year period. Ann. Pharmacother. 48, 954-961. doi: 10.1177/1060028014535363

Gadzhanova, S., Roughead, E., and Robinson, M. (2015). Use of medicines with anticholinergic and sedative effect before and after initiation of anti-dementia medications. Drugs Real World Outcomes 2, 53-60. doi: 10.1007/s40801-0150012-y

Gnjidic, D., Hilmer, S. N., Hartikainen, S., Tolppanen, A. M., Taipale, H., Koponen, M., et al. (2014). Impact of high risk drug use on hospitalization and mortality in older people with and without Alzheimer's disease: a national population cohort study. PLoS One 9:e83224. doi: 10.1371/journal.pone.0083224

Gnjidic, D., Le Couteur, D. G., Naganathan, V., Cumming, R. G., Creasey, H., Waite, L. M., et al. (2012). Effects of drug burden index on cognitive function in older men. J. Clin. Psycho. Pharmacol. 32, 273-277. doi: 10.1097/JCP. 0b013e3182487825

Gorup, E., Rifel, J., and PetekSter, M. (2018). Anticholinergic burden and most common anticholinergic-acting medicines in older general practice patients. ZdrVarst. 57, 140-147. doi: 10.2478/sjph-2018-0018

Grande, G., Tramacere, I., Vetrano, D. L., Clerici, F., Pomati, S., Mariani, C., et al. (2017). REMIND Study Group (Rete Milanese Integrata per le Demenze). Role of anticholinergic burden in primary care patients with first cognitive complaints. Eur. J. Neurol. 24, 950-955. doi: 10.1111/ene.13313

Gray, S. L., Anderson, M. L., Dublin, S., Hanlon, J. T., Hubbard, R., Walker, R., et al. (2015). Cumulative use of strong anticholinergics and incident dementia: a prospective cohort study. JAMA Intern. Med. 175, 401-407. doi: 10.1001/ jamainternmed.2014.7663

Gray, S. L., Anderson, M. L., Hanlon, J. T., Dublin, S., Walker, R. L., Hubbard, R. A., et al. (2018). Exposure to strong anticholinergic medications and dementiarelated neuropathology in a community-based autopsy cohort. J. Alzheimers Dis. 65, 607-616. doi: 10.3233/JAD-171174

Green, A. R., Oh, E., Hilson, L., Tian, J., and Boyd, C. M. (2016). Anticholinergic burden in older adults with mild cognitive impairment. J. Am. Geriatr. Soc. 64, e313-e314. doi: 10.1111/jgs.14554

Han, L., Agostini, J. V., and Allore, H. G. (2008). Cumulative anticholinergic exposure is associated with poor memory and executive function in older men. J. Am. Geriatr. Soc. 56, 2203-2210. doi: 10.1111/j.1532-5415.2008.02009.x

Han, L., McCusker, J., Cole, M., Abrahamowicz, M., Primeau, F., and Elie, M. (2001). Use of medications with anticholinergic effect predicts clinical severity of delirium symptoms in older medical inpatients. Arch. Intern. Med. 161, 1099-1105.

Hilmer, S. N., Mager, D. E., Simonsick, E. M., Cao, Y., Ling, S. M., Windham, B. G., et al. (2007). A drug burden index to define the functional burden of medications in older people. Arch. Intern. Med. 167, 781-787.

Hori, K., Konishi, K., Hosoi, M., Tomioka, H., Tani, M., Kitajima, Y., et al. (2016). Mini review: anticholinergic activity as a behavioral pathology of lewy body disease and proposal of the concept of "Anticholinergic Spectrum Disorders". Parkinsons. Dis. 2016:5380202.

Hori, K., Konishi, K., Watanabe, K., Uchida, H., Tsuboi, T., Moriyasu, M., et al. (2011). Influence of anticholinergic activity in serum on clinical symptoms of Alzheimer's disease. Neuropsychobiology 63, 147-153. doi: 10.1159/000321591

Jamsen, K. M., Gnjidic, D., Hilmer, S. N., Ilomäki, J., Le Couteur, D. G., Blyth, F. M., et al. (2017). Drug burden index and change in cognition over time in community-dwelling older men: the CHAMP study. Ann. Med. 49, 157-164. doi: $10.1080 / 07853890.2016 .1252053$

Jessen, F., Kaduszkiewicz, H., Daerr, M., Bickel, H., Pentzek, M., Riedel-Heller, S., et al. (2010). Anticholinergic drug use and risk for dementia: target for dementia prevention. Eur. Arch. Psychiatry Clin. Neurosci. 260(Suppl. 2), S111-S115. doi: 10.1007/s00406-010-0156-4
Kachru, N., Carnahan, R. M., Johnson, M. L., and Aparasu, R. R. (2015). Potentially inappropriate anticholinergic medication use in older adults with dementia. J. Am. Pharm. Assoc. 55, 603-612. doi: 10.1331/JAPhA.2015.14288

Kersten, H., Molden, E., Tolo, I. K., Skovlund, E., Engedal, K., and Wyller, T. B. (2013). Cognitive effects of reducing anticholinergic drug burden in a frail elderly population: a randomized controlled trial. J. Gerontol. A Biol. Sci. Med. Sci. 68, 271-278. doi: 10.1093/gerona/gls176

Kitajima, Y., Hori, K., Konishi, K., Tani, M., Tomioka, H., Akashi, N., et al. (2015). A review of the role of anticholinergic activity in lewy body disease and delirium. Neurodegener. Dis. 15, 162-167. doi: 10.1159/000381522

Konishi, K., Hori, K., Tani, M., Tomioka, H., Kitajima, Y., Akashi, N., et al. (2015). Hypothesis of endogenous anticholinergic activity in Alzheimer's disease Neurodegener. Dis. 15, 149-156. doi: 10.1159/000381511

Konishi, K., Hori, K., Uchida, H., Watanabe, K., Tominaga, I., Kimura, M., et al. (2010). Adverse effects of anticholinergic activity on cognitive functions in Alzheimer's disease. Psychogeriatrics 10, 34-38. doi: 10.1111/j.1479-8301.2010. 00310.x

Kose, E., Hirai, T., Seki, T., Hidaka, S., and Hamamoto, T. (2018). Anticholinergic load negatively correlates with recovery of cognitive activities of daily living for geriatric patients after stroke in the convalescent stage. J. Clin. Pharm. Ther. 43, 799-806. doi: 10.1111/jcpt.12706

Kröger, E., Simard, M., Sirois, M. J., Giroux, M., Sirois, C., Kouladjian-O’Donnell, L., et al. (2019). Is the drug burden index related to declining functional status at follow-up in community-dwelling seniors consulting for minor injuries? Results from the Canadian emergency team initiative cohort study. Drugs Aging 36, 73-83. doi: 10.1007/s40266-018-0604-9

Lai, M. K., Lai, O. F., Keene, J., Esiri, M. M., Francis, P. T., Hope, T., et al. (2001). Psychosis of Alzheimer's disease is associated with elevated muscarinic M2 binding in the cortex. Neurology 57, 805-811. doi: 10.1212/wnl.57.5.805

Lattanzio, F., Corica, F., Schepisi, R., Amantea, D., Bruno, F., Cozza, A., et al. (2018). Anticholinergic burden and 1-year mortality among older patients discharged from acute care hospital. GeriatrGerontol. Int. 18, 705-713. doi: 10.1111 /ggi.13234

Lechevallier-Michel, N., Molimard, M., Dartigues, J. F., Fabrigoule, C., and Fourrier-Réglat, A. (2005). Drugs with anticholinergic properties and cognitive performance in the elderly: results from the PAQUID study. Br. J. Clin. Pharmacol. 59, 143-151. doi: 10.1111/j.1365-2125.2004.02232.x

Lemstra, A. W., Eikelenboom, P., and van Gool, W. A. (2003). The cholinergic deficiency syndrome and its therapeutic implications. Gerontology 49, 55-60. doi: $10.1159 / 000066508$

Limback-Stokin, M. M., Krell-Roesch, J., Roesler, K., Hansen, A., Stonnington, C. M., Temkit, M., et al. (2018). Anticholinergic medications and cognitive function in late midlife. Alzheimer Dis. Assoc. Disord. 32, 262-264. doi: 10.1097/ WAD. 0000000000000251

López-Álvarez, J., and Agüera Ortiz, L. (2019). [STOPP/START criteria and psycho-geriatric practice]. Rev. EspGeriatr. Gerontol. 54:61. doi: 10.1016/j.regg. 2018.07.005

Lowry, E., Woodman, R. J., Soiza, R. L., and Mangoni, A. A. (2011). Associations between the anticholinergic risk scale score and physical function: potential implications for adverse outcomes in older hospitalized patients. J. Am. Med. Dir. Assoc. 12, 565-572. doi: 10.1016/j.jamda.2011.03.006

Lu, C. J., and Tune, L. E. (2003). Chronic exposure to anticholinergic medications adversely affects the course of Alzheimer disease. Am. J. Geriatr. Psychiatry 11, 458-461. doi: 10.1176/appi.ajgp.11.4.458

Lupu, A. M., Clinebell, K., Gannon, J. M., Ellison, J. C., and Chengappa, K. N. R. (2017). Reducing anticholinergic medication burden in patients with psychotic or bipolar disorders. J. Clin. Psychiatry 78, e1270-e1275. doi: 10.4088/JCP. $16 \mathrm{~m} 11269$

Mangoni, A. A., van Munster, B. C., Woodman, R. J., and de Rooij, S. E. (2013). Measures of anticholinergic drug exposure, serum anticholinergic activity, and all-cause postdischarge mortality in older hospitalized patients with hip fractures. Am. J. Geriatr. Psychiatry 21, 785-793. doi: 10.1016/j.jagp.2013.01. 012

Mantri, S., Fullard, M., Gray, S. L., Weintraub, D., Hubbard, R. A., Hennessy, S., et al. (2019). Patterns of Dementia treatment and frank prescribing errors in older adults with Parkinson disease. JAMA Neurol. 76, 41-49. doi: 10.1001/ jamaneurol.2018.2820 
Marcum, Z. A., Perera, S., Thorpe, J. M., Switzer, G. E., Gray, S. L., Castle, N. G., et al. (2015). Health ABC Study, USA. Anticholinergic use and recurrent falls in community-dwelling older adults: findings from the health $\mathrm{ABC}$ study. Ann. Pharmacother. 49, 1214-1221. doi: 10.1177/1060028015596998

Maust, D. T., Kim, H. M., Seyfried, L. S., Chiang, C., Kavanagh, J., Schneider, L. S., et al. (2015). Antipsychotics, other psychotropics, and the risk of death in patients with dementia: number needed to harm. JAMA Psychiatry 72, 438-445. doi: 10.1001/jamapsychiatry.2014.3018

Mayer, T., Kopitz, J., Plaschke, K., Weiss, J., Seidling, H. M., and Haefeli, W. E. (2016). Limitations of the anticholinergic activity assay and assay-based anticholinergic drug scales. Am. J. Geriatr. Psychiatry 24, 1182-1188. doi: 10. 1016/j.jagp.2016.07.024

Mayer, T., Meid, A. D., Saum, K. U., Brenner, H., Schöttker, B., Seidling, H. M., et al. (2017). Comparison of nine instruments to calculate anticholinergic load in a large cohort of older outpatients: association with cognitive and functional decline, falls, and use of laxatives. Am. J. Geriatr. Psychiatry 25, 531-540. doi: 10.1016/j.jagp.2017.01.009

Moore, A. R., and O'Keeffe, S. T. (1999). Drug-induced cognitive impairment in the elderly. Drugs Aging 15, 15-28. doi: 10.2165/00002512-199915010-00002

Moorey, H. C., Zaidman, S., and Jackson, T. A. (2016). Delirium is not associated with anticholinergic burden or polypharmacy in older patients on admission to an acute hospital: an observational case control study. BMC Geriatr. 16:162. doi: 10.1186/s12877-016-0336-9

Morrow, S. A., Rosehart, H., Sener, A., and Welk, B. (2018). Anti-cholinergic medications for bladder dysfunction worsen cognition in persons with multiple sclerosis. J. Neurol. Sci. 385, 39-44. doi: 10.1016/j.jns.2017.11.028

Mulsant, B. H., Pollock, B. G., Kirshner, M., Shen, C., Dodge, H., and Ganguli, M. (2003). Serum anticholinergic activity in a community-based sample of older adults: relationship with cognitive performance. Arch. Gen. Psychiatry 60, 198-203.

Nakra, B. R., Margolis, R. B., Gfeller, J. D., Grossberg, G. T., and Sata, L. S. (1992). The effect of a single low dose of trihexyphenidyl on memory functioning in the healthy elderly. Int. Psychogeriatr. Fall 4, 207-214. doi: 10.1017/ s1041610292001030

Naples, J. G., Marcum, Z. A., Perera, S., Gray, S. L., Newman, A. B., Simonsick, E. M., et al. (2015). Health, aging and body composition study. Concordance between anticholinergic burden scales. J. Am. Geriatr. Soc. 63, 2120-2124. doi: 10.1111 /jgs. 13647

Nishiyama, K., Sugishita, M., Kurisaki, H., and Sakuta, M. (1998) Reversible memory disturbance and intelligence impairment induced by long-term anticholinergic therapy. Intern. Med. 37, 514-518. doi: 10.2169/internalmedicine.37.514

Niznik, J., Zhao, X., Jiang, T., Hanlon, J. T., Aspinall, S. L., Thorpe, J., et al. (2017) Anticholinergic prescribing in medicare part $\mathrm{D}$ beneficiaries residing in nursing homes: results from a retrospective cross-sectional analysis of medicare data. Drugs Aging 34, 925-939. doi: 10.1007/s40266-017-0502-6

O’Mahony, D., O’Sullivan, D., Byrne, S., O'Connor, M. N., Ryan, C., and Gallagher, P. (2015). STOPP/START criteria for potentially inappropriate prescribing in older people: version 2. Age Ageing 44, 213-218. doi: 10.1093/ageing/af u145

O’Reilly, K., O’Connell, P., Donohoe, G., Coyle, C., O’Sullivan, D., Azvee, Z., et al. (2016). Anticholinergic burden in schizophrenia and ability to benefit from psychosocial treatment programmes: a 3-year prospective cohort study. Psychol. Med. 46, 3199-3211. doi: 10.1017/s0033291716002154

Pasina, L., Colzani, L., Cortesi, L., Tettamanti, M., Zambon, A., Nobili, A., et al. (2019). Relation between Delirium and Anticholinergic drug burden in a cohort of hospitalized older patients: an observational study. Drugs Aging 36, 85-91. doi: $10.1007 / \mathrm{s} 40266-018-0612-9$

Pasina, L., Djade, C. D., Lucca, U., Nobili, A., Tettamanti, M., Franchi, C., et al. (2013). Association of anticholinergic burden with cognitive and functional status in a cohort of hospitalized elderly: comparison of the anticholinergic cognitive burden scale and anticholinergic risk scale: results from the REPOSI study. Drugs Aging 30, 103-112. doi: 10.1007/s40266-012-0044-x

Perry, E. K., Kilford, L., Lees, A. J., Burn, D. J., and Perry, R. H. (2003). Increased Alzheimer pathology in Parkinson's disease related to antimuscarinic drugs. Ann. Neurol. 54, 235-238. doi: 10.1002/ana.10639

Pfistermeister, B., Tümra, T., Gaßmann, K. G., Maas, R., and Fromm, M. F. (2017). Anticholinergic burden and cognitive function in a large German cohort of hospitalized geriatric patients. PLoS One 12:e0171353. doi: 10.1371/journal. pone. 0171353

Plaschke, K., Kopitz, J., Mattern, J., Martin, E., and Teschendorf, P. (2010) Increased cortisol levels and anticholinergic activity in cognitively unimpaired patients. J. Neuropsychiatry Clin. Neurosci. Fall 22, 433-441. doi: 10.1176/appi. neuropsych.22.4.433

Plaschke, K., Petersen, K. A., Frankenhauser, S., Weigand, M. A., Kopitz, J., and Bardenheuer, H. J. (2016). The impact of plasma cholinergic enzyme activity and other risk factors for the development of Delirium in patients receiving palliative care. J. Pain Symptom Manag. 52, 525-532. doi: 10.1016/j. jpainsymman.2016.05.015

Pomara, N., Willoughby, L. M., Wesnes, K., and Sidtis, J. J. (2004). Increased anticholinergic challenge-induced memory impairment associated with the APOE-epsilon4 allele in the elderly: a controlled pilot study. Neuropsychopharmacology 29, 403-409. doi: 10.1038/sj.npp.1300305

Pont, L. G., Nielen, J. T., McLachlan, A. J., Gnjidic, D., Chan, L., Cumming, R. G. et al. (2015). Measuring anticholinergic drug exposure in older communitydwelling Australian men: a comparison of four different measures. Br. J. Clin. Pharmacol. 80, 1169-1175. doi: 10.1111/bcp.12670

Potamianos, G., and Kellett, J. M. (1982). Anti-cholinergic drugs and memory: the effects of benzhexol on memory in a group of geriatric patients. Br. J. Psychiatry 140, 470-472. doi: 10.1192/bjp.140.5.470

Rehse, M., Bartolovic, M., Baum, K., Richter, D., Weisbrod, M., and RoeschEly, D. (2016). Influence of antipsychotic and anticholinergic loads on cognitive functions in patients with Schizophrenia. Schizophr. Res. Treatment 2016:8213165. doi: 10.1155/2016/8213165

Richardson, K., Fox, C., Maidment, I., Steel, N., Loke, Y. K., Arthur, A., et al. (2018). Anticholinergic drugs and risk of dementia: case-control study. BMJ 361:k1315. doi: 10.1136/bmj.k1315

Richter, N., Allendorf, I., Onur, O. A., Kracht, L., Dietlein, M., Tittgemeyer, M., et al. (2014). The integrity of the cholinergic system determines memory performance in healthy elderly. Neuroimage 100, 481-488. doi: 10.1016/j. neuroimage.2014.06.031

Risacher, S. L., McDonald, B. C., Tallman, E. F., West, J. D., Farlow, M. R., Unverzagt, F. W., et al. (2016). Alzheimer's disease neuroimaging initiative. Association between anticholinergic medication use and cognition, brain metabolism, and brain atrophy in cognitively normal older adults. JAMA Neurol. 73, 721-732. doi: 10.1001/jamaneurol.2016.0580

Roe, C. M., Anderson, M. J., and Spivack, B. (2002). Use of anticholinergic medications by older adults with dementia. J. Am. Geriatr. Soc. 50, 836-842. doi: 10.1046/j.1532-5415.2002.50208.x

Rudolph, J. L., Salow, M. J., Angelini, M. C., and McGlinchey, R. E. (2008). The anticholinergic risk scale and anticholinergic adverse effects in older persons. Arch. Intern. Med. 168, 508-513. doi: 10.1001/archinternmed.2007.106

Ruxton, K., Woodman, R. J., and Mangoni, A. A. (2015). Drugs with anticholinergic effects and cognitive impairment, falls and all-cause mortality in older adults: a systematic review and meta-analysis. Br. J. Clin. Pharmacol. 80, 209-220. doi: 10.1111/bcp.12617

Salahudeen, M. S., Chyou, T. Y., and Nishtala, P. S. (2016). Serum Anticholinergic activity and cognitive and functional adverse outcomes in older people: a systematic review and meta-analysis of the literature. PLoS One 11:e0151084. doi: 10.1371/journal.pone.0151084

Salahudeen, M. S., Duffull, S. B., and Nishtala, P. S. (2014). Impact of anticholinergic discontinuation on cognitive outcomes in older people: a systematic review. Drugs Aging 31, 185-192. doi: 10.1007/s40266-014-0158-4

Salahudeen, M. S., Duffull, S. B., and Nishtala, P. S. (2015). Anticholinergic burden quantified by anticholinergic risk scales and adverse outcomes in older people: a systematic review. BMC Geriatr. 15:31. doi: 10.1186/s12877-015-0029-9

Salahudeen, M. S., and Nishtala, P. S. (2016). Examination and estimation of anticholinergic burden: current trends and implications for future research. Drugs Aging 33, 305-313. doi: 10.1007/s40266-016-0362-5

Sanders, L. M. J., Hortobágyi, T., van Staveren, G., Taxis, K., Boersma, F., Klein, H. C., et al. (2017). Relationship between drug burden and physical and cognitive functions in a sample of nursing home patients with dementia. Eur. J. Clin. Pharmacol. 73, 1633-1642. doi: 10.1007/s00228-017-2319-y

Sathienluckana, T., Unaharassamee, W., Suthisisang, C., Suanchang, O., and Suansanae, T. (2018). Anticholinergic discontinuation and cognitive functions in patients with schizophrenia: a pharmacist-physician collaboration in the 
outpatient department. Integr. Pharm Res. Pract. 26, 161-171. doi: 10.2147/ IPRP.S176653

Schreiber, S. R., Wojtalik, J. A., Walker, C. P., Cho, R. Y., Eack, S. M., and Keshavan, M. S. (2018). Serum anticholinergicity is associated with reduced prefrontal brain function in early course schizophrenia. Psychiatry Res. Neuroimaging 281, 31-34. doi: 10.1016/j.pscychresns.2018.08.017

Sink, K. M., Thomas, J. III, Xu, H., Craig, B., Kritchevsky, S., and Sands, L. P. (2008). Dual use of bladder anticholinergics and cholinesterase inhibitors: longterm functional and cognitive outcomes. J. Am. Geriatr. Soc. 56, 847-853. doi: 10.1111/j.1532-5415.2008.01681.x

Sittironnarit, G., Ames, D., Bush, A. I., Faux, N., Flicker, L., Foster, J., et al. (2011). AIBL research group. Effects of anticholinergic drugs on cognitive function in older Australians: results from the AIBL study. Dement. Geriatr. Cogn. Disord. 31, 173-178. doi: 10.1159/000325171

Sperling, R., Greve, D., Dale, A., Killiany, R., Holmes, J., Rosas, H. D., et al. (2002). Functional MRI detection of pharmacologically induced memory impairment. Proc. Natl. Acad. Sci. U.S.A. 99, 455-460. doi: 10.1073/pnas.012467899

Sverdrup Efjestad, A., Ihle-Hansen, H., Hjellvik, V., and Blix, H. S. (2017). Comedication and treatment length in users of acetylcholinesterase inhibitors. Dement. Geriatr. Cogn. Dis. Extra. 7, 30-40. doi: 10.1159/000454948

Swami, S., Cohen, R. A., Kairalla, J. A., and Manini, T. M. (2016). Anticholinergic drug use and risk to cognitive performance in older adults with questionable cognitive impairment: a cross-sectional analysis. Drugs Aging 33, 809-818. doi: 10.1007/s40266-016-0400-3

Tan, E. C. K., Eriksdotter, M., Garcia-Ptacek, S., Fastbom, J., and Johnell, K. (2018). Anticholinergic burden and risk of stroke and death in people with different types of Dementia. J Alzheimers Dis. 65, 589-596. doi: 10.3233/JAD- 180353

Teramura-Grönblad, M., Muurinen, S., Soini, H., Suominen, M., and Pitkälä, K. H. (2011). Use of anticholinergic drugs and cholinesterase inhibitors and their association with psychological well-being among frail older adults in residential care facilities. Ann. Pharmacother. 45, 596-602. doi: 10.1345/aph.1P650

Tsoutsoulas, C., Mulsant, B. H., Kumar, S., Ghazala, Z., Voineskos, A. N., Menon, M., et al. (2017). Anticholinergic Burden and cognition in older patients with Schizophrenia. J. Clin. Psychiatry 78, e1284-e1290. doi: 10.4088/JCP.17m11523

Tune, L., and Coyle, J. T. (1980). Serum levels of anticholinergic drugs in treatment of acute extrapyramidal side effects. Arch. Gen. Psychiatry 37, 293-297.

Uusvaara, J., Pitkala, K. H., Tienari, P. J., Kautiainen, H., Tilvis, R. S., and Strandberg, T. E. (2009). Association between anticholinergic drugs and apolipoprotein E epsilon4 allele and poorer cognitive function in older cardiovascular patients: a cross-sectional study. J. Am. Geriatr. Soc. 57, 427-431. doi: 10.1111/j.1532-5415.2008.02129.x

Vetrano, D. L., La Carpia, D., Grande, G., Casucci, P., Bacelli, T., Bernabei, R., et al. (2016). Italian Group for appropriate drug prescription in the elderly (IGrADE). Anticholinergic medication burden and 5-year risk of hospitalization and death in nursing home elderly residents with coronary artery disease. J. Am. Med. Dir. Assoc. 17, 1056-1059. doi: 10.1016/j.jamda.2016.07.012

Vingerhoets, C., Bakker, G., van Dijk, J., Bloemen, O. J. N., Wang, Y., Chan, R. C. K., et al. (2017). The effect of the muscarinic M1 receptor antagonist biperiden on cognition in medication free subjects with psychosis. Eur. Neuropsychopharmacol. 27, 854-864. doi: 10.1016/j.euroneuro.2017.06.014

Wawruch, M., Macugova, A., Kostkova, L., Luha, J., Dukat, A., Murin, J., et al. (2012). The use of medications with anticholinergic properties and risk factors for their use in hospitalised elderly patients. Pharmacoepidemiol. Drug Saf. 21, 170-176. doi: 10.1002/pds.2169
Weichert, I., Romero-Ortuno, R., Tolonen, J., Soe, T., Lebus, C., Choudhury, S., et al. (2018). Global research on acute conditions team (GREAT). Anticholinergicmedications in patients admitted with cognitive impairment or falls (AMiCI). The impact of hospital admission on anticholinergic cognitive medication burden. Results of a multicentre observational study. J. Clin. Pharm Ther. 43, 682-694. doi: 10.1111/jcpt. 12694

Whalley, L. J., Sharma, S., Fox, H. C., Murray, A. D., Staff, R. T., Duthie, A. C., et al. (2012). Anticholinergic drugs in late life: adverse effects on cognition but not on progress to dementia. J. Alzheimers Dis. 30, 253-261. doi: 10.3233/JAD-2012110935

Williams, A., Sera, L., and McPherson, M. L. (2018). Anticholinergic burden in hospice patients with Dementia. Am. J. Hosp. Palliat. Care 13, 1049909118800281. doi: 10.1177/1049909118800281

Wilson, N. M., Hilmer, S. N., March, L. M., Cameron, I. D., Lord, S. R., Seibel, M. J., et al. (2011). Associations between drug burden index and falls in older people in residential aged care. J. Am. Geriatr. Soc. 59, 875-880. doi: 10.1111/j.15325415.2011.03386.x

Wolters, A. E., Zaal, I. J., Veldhuijzen, D. S., Cremer, O. L., Devlin, J. W., van Dijk, D., et al. (2015). Anticholinergic medication use and transition to Delirium in critically ill patients: a prospective cohort study. Crit. Care Med. 43, 1846-1852. doi: 10.1097/CCM.0000000000001094

Wouters, H., van der Meer, H., and Taxis, K. (2017). Quantification of anticholinergic and sedative drug load with the DrugBurdenIndex: a review of outcomes and methodological quality of studies. Eur. J. Clin. Pharmacol. 73, 257-266. doi: 10.1007/s00228-016-2162-6

Wu, Y. H., Wang, C. J., Hung, C. H., Chen, L. Y., Lin, M. H., Wang, P. N., et al. (2017). Association between using medications with anticholinergic properties and short-term cognitive decline among older men: a retrospective cohort study in Taiwan. Geriatr. Gerontol. Int. 17(Suppl. 1), 57-64. doi: 10.1111/ggi. 13032

Yoshiyama, Y., Kojima, A., Itoh, K., Isose, S., Koide, M., Hori, K., et al. (2015). Does anticholinergic activity affect neuropathology? implication of neuroinflammation in Alzheimer's disease. Neurodegener. Dis. 15, 140-148. doi: $10.1159 / 000381484$

Zia, A., Kamaruzzaman, S., Myint, P. K., and Tan, M. P. (2016). Anticholinergic burden is associated with recurrent and injurious falls in older individuals. Maturitas 84, 32-37. doi: 10.1016/j.maturitas.2015.10.009

Ziad, A., Olekhnovitch, R., Ruiz, F., Berr, C., Bégaud, B., Goldberg, M., et al. (2018). Anticholinergic drug use and cognitive performances in middle age: findings from the CONSTANCES cohort. J. Neurol. Neurosurg. Psychiatry 89, 1107-1115. doi: 10.1136/jnnp-2018-318190

Conflict of Interest: The authors declare that the research was conducted in the absence of any commercial or financial relationships that could be construed as a potential conflict of interest.

Copyright (C) 2019 López-Álvarez, Sevilla-Llewellyn-Jones and Agüera-Ortiz. This is an open-access article distributed under the terms of the Creative Commons Attribution License (CC BY). The use, distribution or reproduction in other forums is permitted, provided the original author(s) and the copyright owner(s) are credited and that the original publication in this journal is cited, in accordance with accepted academic practice. No use, distribution or reproduction is permitted which does not comply with these terms. 